\title{
IMPLEMENTATION OF AN ADAPTIVE ARTIFICIAL VISION SYSTEM APPLIED TO VIDEO SURVEILLANCE TASKS ON RASPBERRY PI
}

\section{MIGUEL PÉREZ. P. \& HOLMAN MONTIEL. A}

Facultad Tecnológica, Universidad Distrital Francisco José de Caldas, Bogotá, Colombia

Video surveillance tools and systems are a fundamental part of our current society, even more so when there is constant growth both in the technological aspect and in the multiple needs linked to the development of intelligent cities and the various areas of the commercial and productive sectors of a country. This work proposes the implementation of an adaptive artificial vision system applied to video surveillance tasks, making use of a Raspberry PI development card and the OpenCV image processing libraries. The aim is to integrate an adaptive background subtraction algorithm based on the calculation of the median technique as a fundamental basis for the detection and tracking of objects, both in outdoor and indoor environments. The aim of the work is to present a scalable software tool that can be adapted to any type of domotic or IoT application, taking advantage of the multiple expansion possibilities that low-cost development cards such as Raspberry have.
\end{abstract}

KEYWORDS: Background Subtraction, Video Surveillance, Motion Detection, Artificial Vision \& Image Processing

Received: Jun 08, 2020; Accepted: Jun 28, 2020; Published: Sep 03, 2020; Paper Id.: IJMPERDJUN20201043

\section{INTRODUCTION}

The continuous technological development associated with video devices today has become the fundamental pillar of intelligent video surveillance applications [1,2]; with the improved integrated processing capabilities of video cameras, environments or areas of implementation are increasing every day, since events associated with vehicle traffic, security of physical facilities and people, crime control, among many other applications can be easily detected and analyzed without the need for human intervention [3-6][7].

If this type of implementation is analyzed, it can be found that generally these solutions include motion detection and object tracking as a fundamental basis [8].Nowadays there is a great variety of development and application algorithms oriented to image processing and artificial vision [9], it must be taken into account that when developing this type of solutions first the emphasis must be placed on the adequate detection of objects [10,11], to later enter the phases of classification and recognition of information [12-14].

Additionally, the boom of development cards and programming languages (for this case Raspberry and Python), open a wide possibility to integrate this technology in a fast and effective way to the continuous needs and requirements of this digital society; with this we refer to the great demand of devices linked to the Internet of things [15]. The current trend of many users is focused on the acquisition or creation of products that are interconnected through an Internet-based infrastructure [16].

Considering the above, the main objective of this paper is to present the implementation of an adaptive video surveillance system on Raspberry $\mathrm{Pi}$, making use of OpenCV image processing libraries. The proposal developed is based on the use of a conventional HD video USB camera for signal acquisition, in conjunction with 
the use of a background subtraction algorithm for motion detection. It must be considered that this development can be scalable thanks to the multiple expansion possibilities presented by the Raspberry cards, which can go from simple domotics applications to complex developments on IoT.

\section{MATERIALS AND MÉTHODS}

The development of this document initially contemplates the description of the background subtraction algorithm based on the median technique, as the main structure for the motion detection of the proposed video surveillance system. Later, the step-by-step implementation of the Raspberry development system is described so that the solution can be adapted and modified according to the needs of any user with minimal knowledge of the Python programming language.

\section{Background Subtraction}

This is a basic and fundamental technique associated with the detection of moving objects, in which a region of interest or object in the foreground is subtracted from a background or static reference area in the background [17,18]. At present, a great variety of published works have been developed using this technique, which range from descriptive evaluations to comparative studies of the various methodologies applied to this subject [19-21]. A possible classification of the subtraction techniques from a traditional to an advanced point of view can be seen in Table 1 .

Table 1: Background Subtraction Techniques [17]

\begin{tabular}{|l|l|}
\hline \multicolumn{1}{|c|}{ Model } & \multicolumn{1}{|c|}{ Techniques } \\
\hline Basic Models & $\begin{array}{l}\text { Median } \\
\text { Adaptive Median } \\
\text { Approximate Median } \\
\text { Histogram Analysis }\end{array}$ \\
\hline Statistical Models & $\begin{array}{l}\text { Gaussian Model } \\
\text { Gaussian Mixture Model } \\
\text { Mixture of Gaussian } \\
\text { Kernel Density Estimation } \\
\text { Support Vector Machine } \\
\text { Support Vector Regression }\end{array}$ \\
\hline Cluster Models & $\begin{array}{l}\text { K-Means } \\
\text { Codebook }\end{array}$ \\
\hline Non-Parametric Model & Video Background Extractor \\
\hline Hybrid Model & \\
\hline
\end{tabular}

\section{Median Algorithm}

Background subtraction by means of median calculation is a method that introduces the concept of adaptive background models for video images, this method allows to efficiently and effectively differentiate a moving object from a static background using an approximation of the median, it was originally described by McFarlane and Schofield [22]; one of the specific advantages of this technique is that it allows to safely adapt to changes in the scene [23].

The median calculation technique models the background by taking an image of the scene when no object is present and using it as a background model. Objects that are not in this background image can be discriminated by the difference between the background model and the actual image, this difference is made in each frame of the image pixel by pixel.

$$
\mathrm{E}_{(\mathrm{m}, \mathrm{n})}=\left|\mathrm{F}_{(\mathrm{m}, \mathrm{n})}-\mathrm{I}_{(\mathrm{m}, \mathrm{n})}\right|<T \square
$$


Equation (1) estimates a binary label $E_{(m, n)}$ by the absolute difference between the background image $F_{(m, n)}$ and the current image $I_{(m, n)}$. If the absolute value of the difference is below the threshold, ThIn the case of the background, the pixel is classified as background, this solution is widely used for scenes that take place in indoor environments and that allow capturing the background without the presence of the objects to be detected. The sub-index ( $m, n)$ refers to the fact that this operation is applied pixel by pixel.

However, the way in which the background image adapts to the varying conditions of the medium and can be executed in algorithms that work in real time without the obligation of having a totally static and object-free background image to detect is by using the adaptive method of median, which is classified as a recursive technique. This technique presents the central number of a set of data ordered in an increasing way as a valid data to model the background and catalogues it as a single mode background model; since it keeps information of the background data for more than half of the last $\mathrm{N}$ frames, the way to calculate the adaptive method of the median and estimate the current background image is shown in equation (2) [24,25].

$$
\mathrm{F}_{(\mathrm{m}, \mathrm{n})}^{\mathrm{t}+1}=\left\{\begin{array}{c}
\mathrm{F}_{(\mathrm{m}, \mathrm{n})}^{\mathrm{t}}+\beta \text { for } \mathrm{I}_{(\mathrm{m}, \mathrm{n})}^{\mathrm{t}+1}>\mathrm{F}_{(\mathrm{m}, \mathrm{n})}^{\mathrm{t}} \\
\mathrm{F}_{(\mathrm{m}, \mathrm{n})}^{\mathrm{t}}-\beta \text { for } \mathrm{I}_{(\mathrm{m}, \mathrm{n})}^{\mathrm{t}+1}<\mathrm{F}_{(\mathrm{m}, \mathrm{n})}^{\mathrm{t}} \\
\mathrm{F}_{(\mathrm{m}, \mathrm{n})}^{\mathrm{t}} \text { for } \mathrm{I}_{(\mathrm{m}, \mathrm{n})}^{\mathrm{t}+1}=\mathrm{F}_{(\mathrm{m}, \mathrm{n})}^{\mathrm{t}}
\end{array}\right.
$$

Where $\mathrm{F}^{\mathrm{t}+1}$ is the estimated background matrix and $\beta$ is a value to adjust the convergence time at which an object becomes the background of the scene, a value of 1 considered high, allows the background to adapt to rapid changes, but in the case of scenes with objects that stop temporarily some other consideration should be made.

\section{IMPLEMENTATION}

Figure 1 shows the block diagram of the application, the program begins by acquiring the image from the Logitech $\mathrm{C} 270$ HD camera which delivers an image in RGB format, this format is susceptible to changes in brightness, so the algorithm applies a format shift to HSB which is more stable for environmental effects.

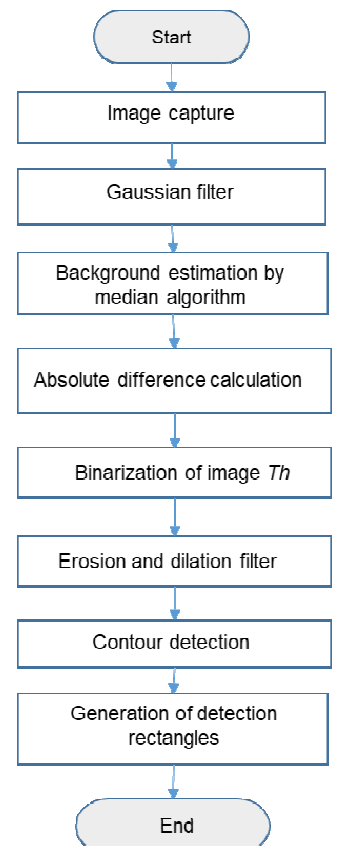

Figure 1: Flow Chart of the Proposed Solution. 
A Gaussian filter is then applied to the image, which is like the media filter and results in decreased sharpness, increased blurring and loss of detail. This filter causes the intensities of small objects to blend with the background and only the larger objects are detected [24], see Figure 2(b). The next step is the application of the algorithm that estimates the background by applying the median technique, as explained in the previous section, this algorithm results in a matrix that represents the current background estimate, see Figure 2(c).

Then, the matrix enters the algorithm that subtracts this estimate with the current image and thus detects all those elements that are not considered as part of the background image, this difference is made based on equation 1, the result is a binary image of the elements that do not belong to the background, see figure 2(d).

However, the binary matrix may have small elements of noise that have not been removed by the Gaussian filter, so the decimation filter is applied. Finally, the algorithm detects the image contour, see figure 2(e), and paints a rectangle in the resulting area of interest, see figure 2(a).

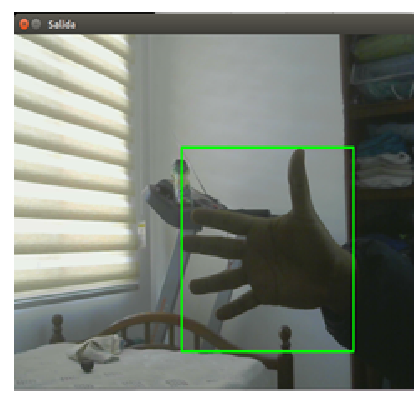

(a)

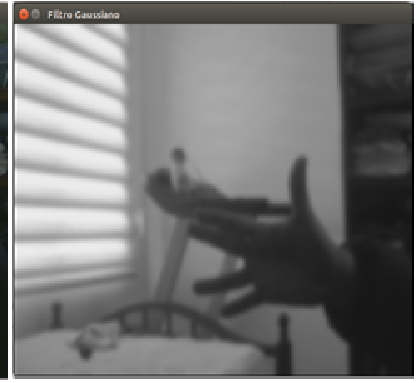

(b)

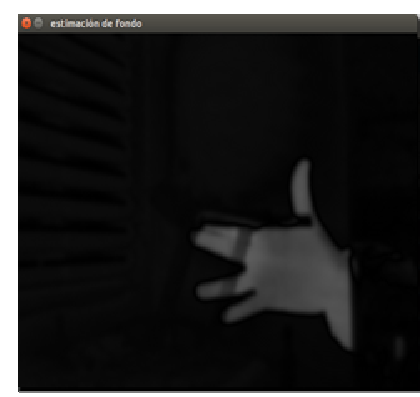

(c)

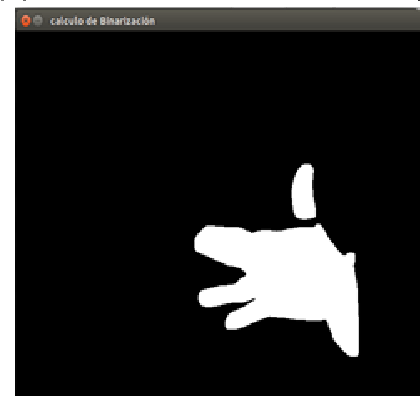

(d)

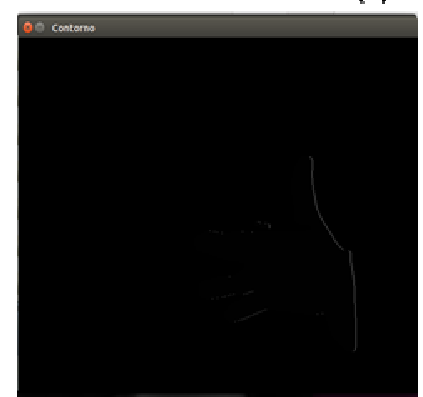

(e)

Figure 2: Debugging and Filtering Processes of the Processed Image. (a) Output Image (b) Gaussian Filter Image (c) Background Estimation (d) Binary Image (e) Contour Detection.

\section{Structure of the source Code}

This section will show in detail the most relevant parts of the implemented system (which are the median method for estimating background and the background subtraction algorithm for foreign object detection), although as mentioned before the system was implemented on a Raspberry PI 3 under Raspbian distribution and Python language 2.7. Algorithm 1 shows the pseudocode of the developed solution, this so that the reader can easily implement it in any programming language.

\footnotetext{
Algorithm 1.Developed application

$F^{t}=I^{t} \#$ the first image is assumed as the background

While true do
} 


$$
\begin{aligned}
& \text { if }{ }^{t+1}{ }_{(\boldsymbol{m}, \boldsymbol{n})}>F_{(m, n)}^{t} \text { then\# Background estimation } \\
& F_{(m, n)}^{t}=F_{(m, n)}^{t-1}+\beta \# \text { by median method } \\
& \text { else if } I_{(\boldsymbol{m}, n)}^{t+1}<F_{(m, n)}^{t} \text { then } \\
& \qquad F_{(m, n)}^{t}=F_{(m, n)}^{t-1}-\beta
\end{aligned}
$$

else

$$
F_{(m, n)}^{t}=F_{(m, n)}^{t}
$$

if $\left|F_{(m, n)}^{t}-I_{(m, n)}^{t+1}\right| \leq T \square$ then \# Calculation of the

$E_{(m, n)}^{t}=1 \#$ binarized label

else

$$
\begin{gathered}
E_{(m, n)}^{t}=0 \\
t=t+1
\end{gathered}
$$

The above algorithm shows the implemented pseudocode, note that $\mathrm{F}^{\mathrm{t}}$ is the background matrix estimated by the algorithm at the current time, which will adapt to the changes in the scene. The matrix $\mathrm{I}^{\mathrm{t}}$ is the image taken by the webcam after the Gaussian filter, the $\beta$ as said before, is the value that adjusts the convergence time in which an object becomes background in the scene, it is important to clarify that each operation is done pixel by pixel so the subscripts $(\mathrm{m}, \mathrm{n})$ in every matrix. Finally, the matrix $\mathrm{E}^{\mathrm{t}}$ is the binary output label, which is the input to the decimation and contour detection filter.

Although the OpenCV library in Python is one of the most complete libraries for image processing it does not have the median technique for background subtraction, so this part of the algorithm was implemented as shown in Algorithm 1. The OpenCV library was used in the two stages of filtering the image (input and output); the input filter that was implemented was a Gaussian filter by means of the code line gris= cv2.GaussianBlur(gris, (21, 21), 0); where grisis the input image in grayscale and the parameters $(21,21)$ is the kernel width that must be positive and odd according to the instructions of the library, a sample of the implemented code can be seen in figure 3 . 


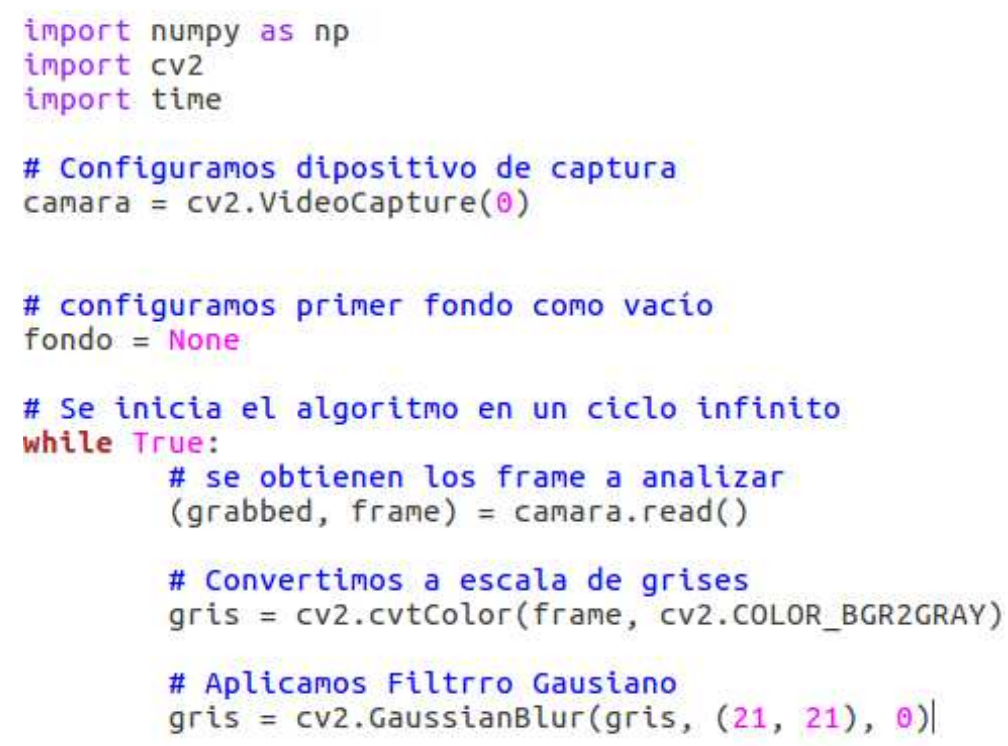

Figure 3: Segments of the Designed Code Associated with Image Capture and Gaussian Filtering using the Open CV Library.

Now, for the system output after calculating the background, two morphological operations belonging to OpenCV were implemented; the first operation is the erosion filter by means of the code line erosion = cv2.erode $($ Label,kernel,iterations $=\mathbf{1})=\mathbf{1})$ that allows to eliminate small elements that are not considered objects of interest, the Label image is the binary output image of the median calculation algorithm and the kernel is the matrix with which the filtering will be done. The second operation is the dilation filter that allows to enlarge the objects of interest; this was done through the code linedilat=cv2.dilate(erosion,kernel,iterations $=\mathbf{1})$, the code implemented in Python can be seen in Figure 4.

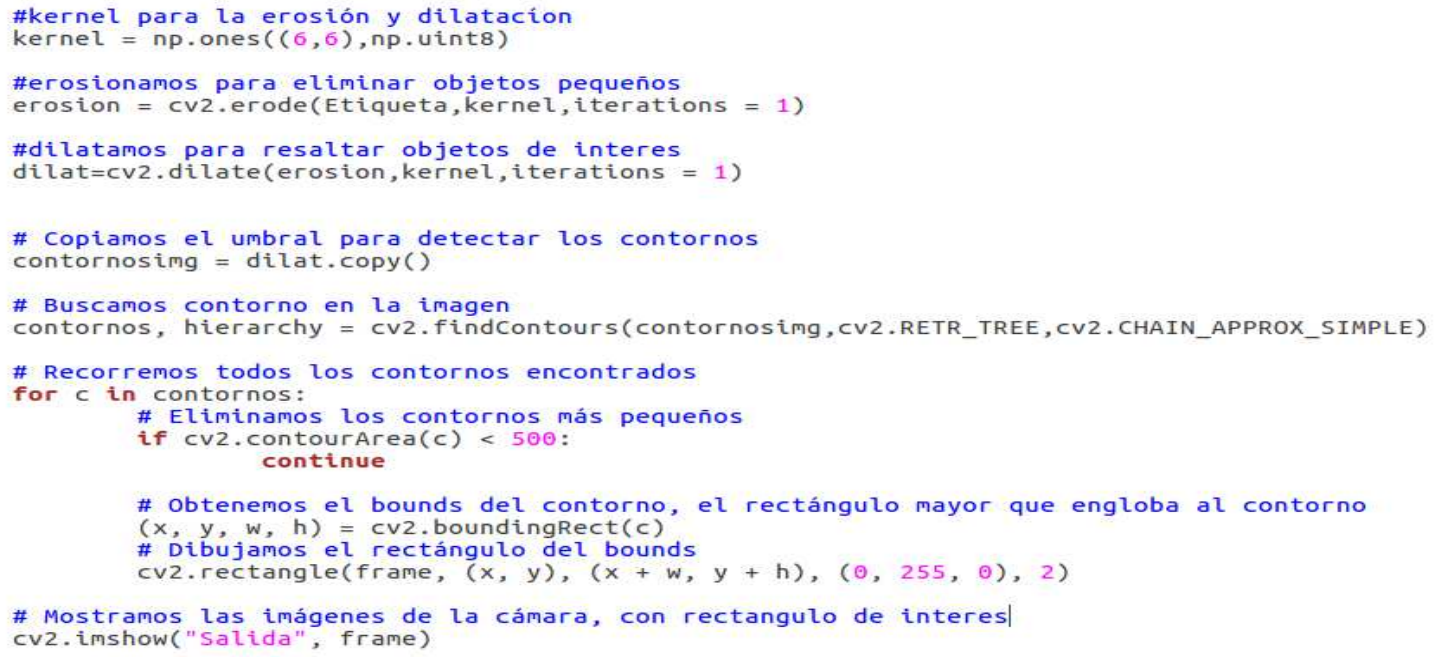

Figure 4: Segments of the Designed Code Associated to the Output Image Filtering by Means of the Open CV Library.

\section{RESULTS}

This section shows the tests that were made on the algorithm in two different environments. The first one in an enclosed space, this environment corresponds to the room of a house with normal lighting, and the second test was done outdoors, in 
an open-air parking lot with natural lighting.

Figure 5 shows the response of the filters and the background estimation algorithm; in the upper part of the figure these responses are observed in an indoor environment, the size of the person to be detected is relatively large due to the proximity of the camera to the scene, facilitating detection, each module performed well in these conditions. However, in the lower part of the figure are shown the response of the filters and the background estimation algorithm in an outdoor environment; note that in this case the person to be detected represents a very small portion of the scene, which would imply a greater complexity for the algorithm, the response of the algorithm is optimal under these conditions.
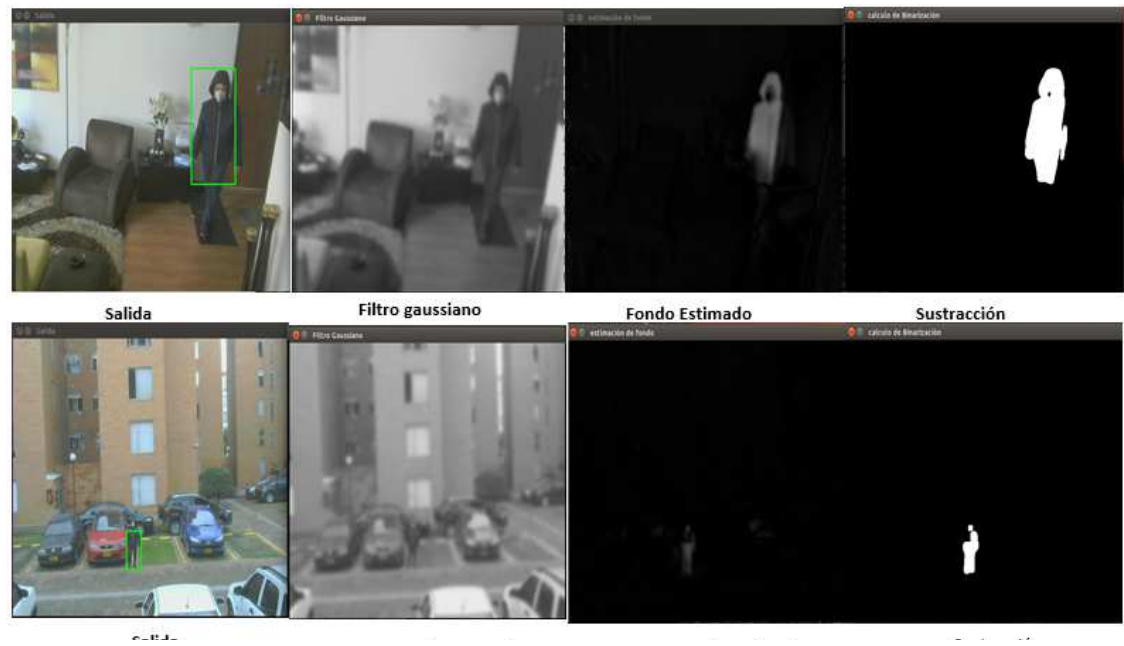

Fondo Estimado Sustracción

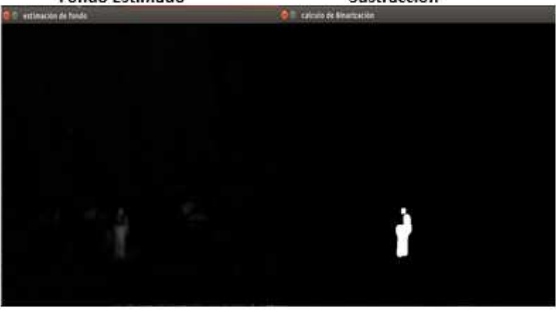

Figure 5: Response of the Different Algorithm Modules in Indoor and Outdoor Space.

Figure 6 shows a successive sequence of images that were obtained from the system in an indoor environment, in this sequence you can see that the algorithm detects more than one object in the scene in some of the frames, these objects are part of the person to detect and is not assumed as false detection, as seen in the images the system behaves optimally in these environments, and its response is ideal for applications in video surveillance.

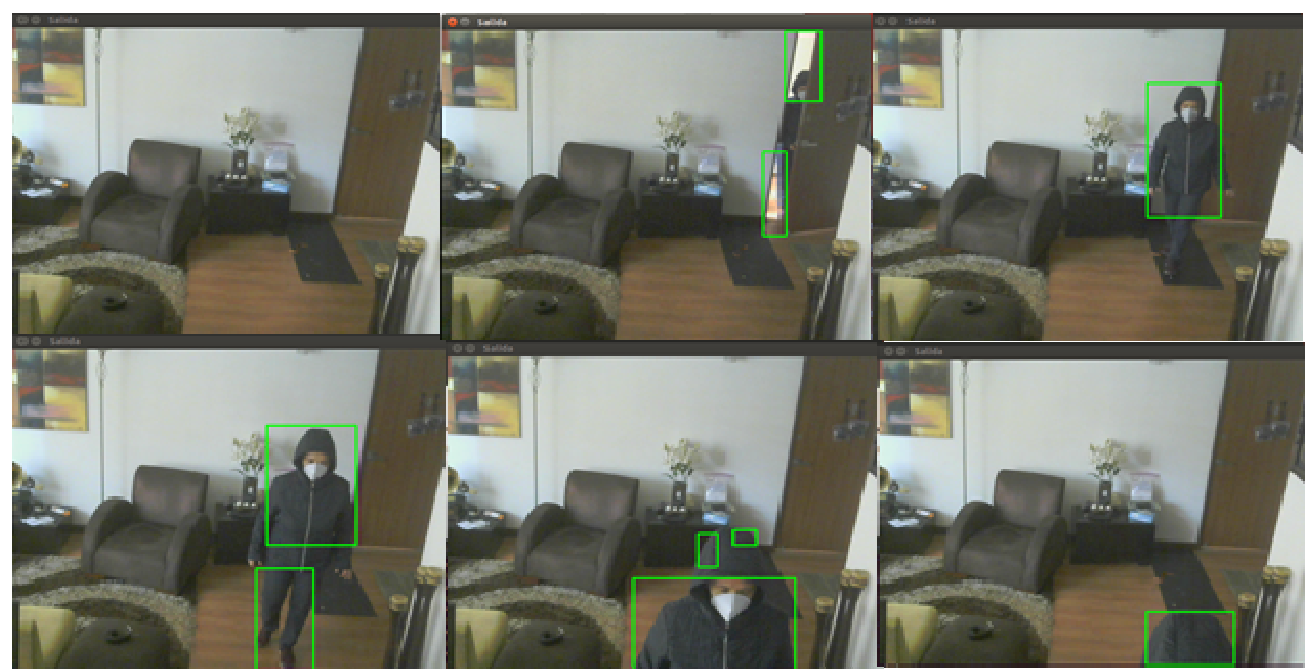

Figure 6: Image Sequence of the Algorithm Output in Indoor Environment.

Figure 7 shows the response of the system in a series of images taken at the output in a successive way, in these images you can see that the scene takes place in an outdoor environment which is subject to natural lighting and therefore variable. It could be observed that the system behaves optimally in this type of environment, which demonstrates the 
efficiency of the median method to adapt the background to changing environments, in addition, it is observed that the system only responds to objects that are moving in the scene, therefore, demonstrates its application for the detection of people in video surveillance applications that take place in outdoor spaces.

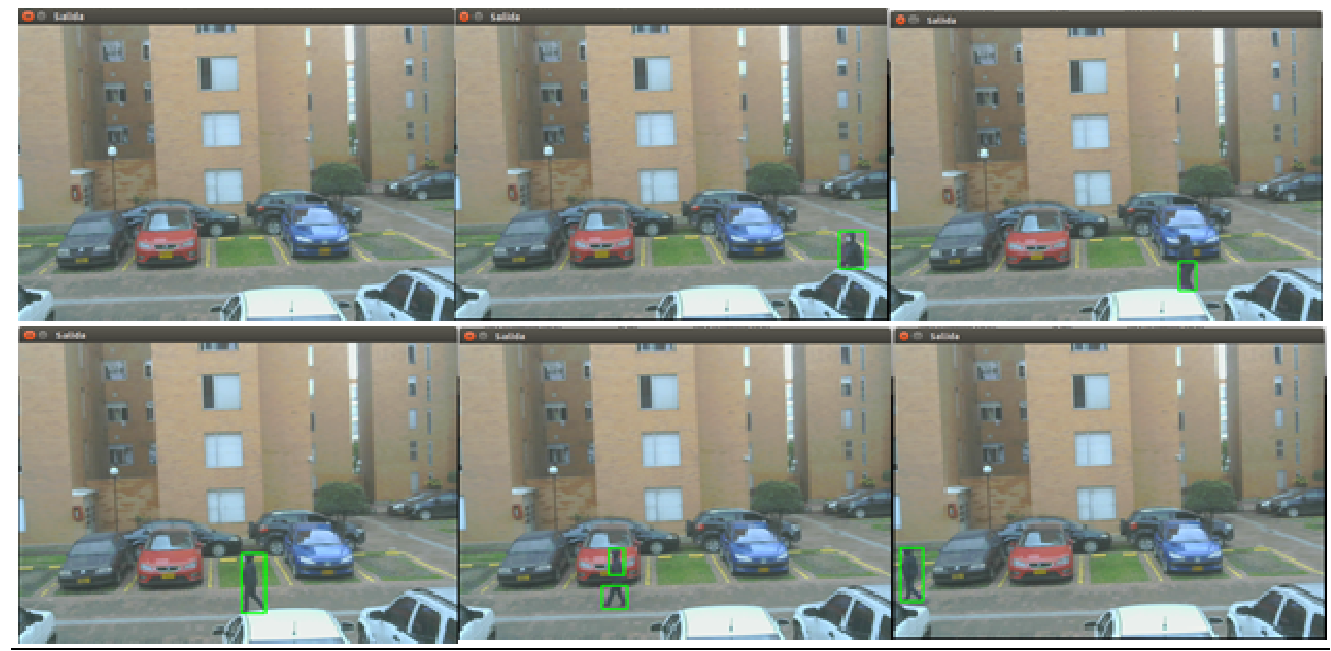

Figure 7: Image Sequence of the Algorithm Output in Indoor Environment.

\section{CONCLUSIONS}

An effective video surveillance tool was developed based on image processing and the adaptation of a background subtraction algorithm, capable of detecting moving objects in outdoor and indoor environments with various variations of lighting. It should be noted that the objective of this implementation was to provide the basis for a possible scalable tool that could be adapted and modified for home automation and/or Internet of Things applications, thanks to the various expansion possibilities that Raspberry development cards have. Although it is true that the total functionality of the implemented algorithm is demonstrated, any developer can incorporate this methodology to adapt it to his needs; a small adaptation of the code would be enough to make the sending of text messages or e-mails, or even, to generate practical solutions of security with the use of additional electronic peripherals like sound or light alarms connected to the GPIO pins of the card.

\section{REFERENCES}

1. Sivabalakrishnan, M., Menaka, R., \& Jeeva, S. (2019). Smart Video Surveillance Systems and Identification of Human Behavior Analysis. Countering Cyber Attacks and Preserving the Integrity and Availability of Critical Systems. 64-97.

2. Kumar, H., Bhattacharya, S., Thomas, S. S., Gupta, S., \& Venkatesh, K. S. (2017). Design of smart video surveillance system for indoor and outdoor scenes. 2017 22nd International Conference on Digital Signal Processing (DSP). 1-5.

3. Beghdadi, A., Asim, M., Almaadeed, N., \& Qureshi, M. A. (2018). Towards the design of smart video-surveillance system. 2018 NASA/ESA Conference on Adaptive Hardware and Systems (AHS). 162-167.

4. Nazare Jr, A. C., \& Schwartz, W. R. (2016). A scalable and flexible framework for smart video surveillance. Computer Vision and Image Understanding, 144, 258-275.

5. Elafi, I., Jedra, M., \& Zahid, N. (2016). Unsupervised detection and tracking of moving objects for video surveillance applications. Pattern Recognit. Lett.,84, 70-77. 
6. Singh, G., Kapoor, R., \& Khosla, A. K. (2019). Intelligent Anomaly Detection Video Surveillance Systems for Smart Cities. In Driving the Development, Management, and Sustainability of Cognitive Cities. 163-182.

7. Mabrouk, A. B., \& Zagrouba, E. (2018). Abnormal behavior recognition for intelligent video surveillance systems: A review. Expert Systems with Applications, 91, 480-491.

8. Supreeth, H.S., \& Patil, C.M. (2018). Efficient multiple moving object detection and tracking using combined background subtraction and clustering. Signal, Image and Video Processing, 12, 1097-1105.

9. Guo, J., Wang, J., Bai, R., Zhang, Y., \& Li, Y. (2017). A new moving object detection method based on frame-difference and background subtraction. IOP Conference Series: Materials Science and Engineering. 242(1).

10. Kuppuswamy, S., \& Panchanathan, B. (2017). Similar Object Detection and Tracking in H.264 Compressed Video Using Modified Local Self Similarity Descriptor and Particle Filtering. International Journal of Intelligent Engineering and Systems, 10, 95-104.

11. Muhammad, K., Ahmad, J., Lv, Z., Bellavista, P., Yang, P. \& Baik, S. (2019). Efficient Deep CNN-Based Fire Detection and Localization in Video Surveillance Applications. IEEE Transactions on Systems, Man, and Cybernetics: Systems, 49(7), 14191434.

12. Darwich, A., Hébert, P. A., Bigand, A., \& Mohanna, Y. (2018). Background subtraction based on a new fuzzy mixture of Gaussians for moving object detection. Journal of Imaging, 4(7), 92.

13. Rea, S., \& Hallo, V. (2018). Autonomous Video Surveillance Application Using Artificial Vision to Track People in Restricted Areas. Developments and Advances in Defense and Security: Proceedings of the Multidisciplinary International Conference of Research Applied to Defense and Security (MICRADS 2018). 94, 58.

14. Figueroa, Y., Arias, L., Mendoza, D., Velasco, N., Rea, S., \& Hallo, V. (2018). Autonomous Video Surveillance Application Using Artificial Vision to Track People in Restricted Areas. International Conference of Research Applied to Defense and Security. 58-68.

15. Khudhair, A. B., \& Ghani, R. F. (2020). IoT Based Smart Video Surveillance System Using Convolutional Neural Network. 2020 6th International Engineering Conference "Sustainable Technology and Development"(IEC). 163-168.

16. Sultana, T., Wahid, K., (2019). Choice of Application Layer Protocols for Next Generation Video Surveillance Using Internet of Video Things. IEEE Access, 7, 41607-41624.

17. Agrawal, S., \& Natu, P. (2020). Segmentation of Moving Objects using Numerous Background Subtraction Methods for Surveillance Applications. International Journal of Innovative Technology and Exploring Engineering (IJITEE), 9(3), 25532563.

18. Maddalena, L., \& Petrosino, A. (2018). Background subtraction for moving object detection in RGBD data: A survey. Journal of Imaging, 4(5), 71.

19. Bayona, Á., SanMiguel, J. C., \& Martínez, J. M. (2009). Comparative evaluation of stationary foreground object detection algorithms based on background subtraction techniques. 2009 Sixth IEEE International Conference on Advanced Video and Signal Based Surveillance. 25-30.

20. Liu, H., Dai, J., Wang, R., Zheng, H., \& Zheng, B. (2016). Combining background subtraction and three-frame difference to detect moving object from underwater video. OCEANS 2016-Shanghai. 1-5.

21. Sobral, A., \& Vacavant, A. (2014). A comprehensive review of background subtraction algorithms evaluated with synthetic and real videos. Computer Vision and Image Understanding, 122, 4-21. 
22. McFarlane, N. J., \& Schofield, C. P. (1995). Segmentation and tracking of piglets in images. Machine vision and applications, 8(3), 187-193.

23. Morales, J. E. S. (2008). Substracción de fondo basado en movimiento (Doctoral dissertation, Centro de Investigación en Matemáticas).

24. Jesus, E. O., \& Costa Jr, R. (2015). A utilização de filtros gaussianos na análise de imagens digitais. Proceeding Series of the Brazilian Society of Computational and Applied Mathematics, 3(1).

25. Bouwmans, T., \& García-García, B. (2019). Visual Surveillance of Human Activities: Background Subtraction Challenges and Methods. From Visual Surveillance to Internet of Things: Technology and Applications, 43. 